\title{
Super-resolution imaging in glycoscience: New developments and challenges
}

\author{
Junling Chen*,†, Ti Tong*,\$,** and Hongda Wang*, ${ }^{*}, * *$ \\ *State Key Laboratory of Electroanalytical Chemistry \\ Changchun Institute of Applied Chemistry \\ Chinese Academy of Sciences \\ Changchun, Jilin 130022, P. R. China \\ ${ }^{\dagger}$ University of Chinese Academy of Sciences \\ Beijing 100049, P. R. China \\ *Department of Thoracic Surgery \\ The Second Hospital of Jilin University Changchun \\ Jilin 130041, P. R. China \\ \$tti666@163.com \\ 『hdwang@ciac.ac.cn
}

Received 29 February 2016

Accepted 6 April 2016

Published 24 May 2016

\begin{abstract}
Carbohydrates on cell surfaces play a crucial role in a wide variety of biological processes, including cell adhesion, recognition and signaling, viral and bacterial infection, inflammation and metastasis. However, owing to the large diversity and complexity of carbohydrate structure and nongenetically synthesis, glycoscience is the least understood field compared with genomics and proteomics. Although the structures and functions of carbohydrates have been investigated by various conventional analysis methods, the distribution and role of carbohydrates in cell membranes remain elusive. This review focuses on the developments and challenges of super-resolution imaging in glycoscience through introduction of imaging principle and the available fluorescent probes for super-resolution imaging, the labeling strategies of carbohydrates, and the recent applications of super-resolution imaging in glycoscience, which will promote the super-resolution imaging technology as a promising tool to provide new insights into the study of glycoscience.
\end{abstract}

Keywords: Carbohydrate; super-resolution imaging.

\section{Introduction}

Carbohydrates, similar to nucleic acids, proteins and lipids, constitute one major class of molecules in living systems. Carbohydrates on cell surfaces carry abundant information about the cell for the interactions with the outside environment or other cells.

${ }^{* *}$ Corresponding authors.

This is an Open Access article published by World Scientific Publishing Company. It is distributed under the terms of the Creative Commons Attribution 4.0 (CC-BY) License. Further distribution of this work is permitted, provided the original work is properly cited. 
With carbohydrates being linked to proteins or lipids, numerous glycoproteins and glycolipids are formed on cell membranes. At the molecular level, carbohydrates in membrane proteins are known to modulate protein solubility, trafficking, and signaling by affecting its folding, structure, and function. ${ }^{1-3}$ In biological systems, carbohydrates also play vital roles in various physiological and pathological processes. The increasing findings indicate that carbohydrates are involved in cell differentiation and development, cell adhesion and motility, recognition, signaling, and immune response. ${ }^{4-6}$ Importantly, alterations in glycosylation are reported to be associated with diverse diseases, including viral and bacterial infection, inflammation, tumorigenesis, cancer differentiation, and metastasis. ${ }^{7-10}$ In spite of ubiquitous distribution and significant functions of carbohydrates, owing to the diversity and complexity of carbohydrate structures, carbohydrates are the least understood molecules among the four major types of biomolecules (nucleic acids, proteins, lipids and carbohydrates).

As an indispensable membrane component, carbohydrates participate in the organization of the plasma membrane, owing to their positions and diverse functions. However, the traditional structure models of the plasma membrane are proposed without appropriately considering the role of carbohydrates, suggesting that the plasma membrane is laterally compartmentalized with various membrane microdomains, and covered by a thick layer of numerous carbohydrates. $^{11-14}$ However, recent findings by Wang et al. ${ }^{15,16}$ imply that the proteins on the ectoplasmic side of cell membranes form a dense protein layer on the top of a lipid bilayer, and the carbohydrates stay in microdomains at the ectoplasmic side to participate in various recognition processes. Besides, glycosylated membrane proteins have been reported to aggregate into microdomains on the plasma membranes via super-resolution imaging. ${ }^{13,17-19}$ Therefore, a deep study of carbohydrates is necessary to reveal the comprehensive organization of plasma membrane.

In the past few years, scientists devoted great effort to develop new methodologies and new tools for isolation, characterization, synthesis, and analysis of carbohydrates. Many kinds of enzymatic treatments were utilized to isolate monosaccharides from the complex carbohydrates, including endoglycosidases, exoglycosidases and potentially destructive chemical cleavage agents (ozone or sodium periodate). Capillary electrophoresis (CE),${ }^{20}$ highperformance anion-exchange chromatography (HPAEC), ${ }^{21}$ and high-performance liquid chromatography (HPLC) ${ }^{22}$ were applied to separate carbohydrates. A series of improvements based on mass spectroscopy (MS) have particular significance in analyzing the sequences and structures of carbohydrates. ${ }^{23}$ Microarray-based technologies have been increasingly applied for comprehensive analysis of glycomics, identification of the glycosylation patterns of glycoproteins, and the interaction of carbohydrate-proteins, including carbohydrate microarrays, lectin microarrays, and glycoprotein microarrays. ${ }^{24-26}$ In spite of obvious advance, these aforementioned methods and tools require prior separation of the attached carbohydrates from their core proteins (in HPLC, CE, MS, NMR, etc.) and are involved in time-consuming analytical and synthetic processes. In fact, a complete understanding of carbohydrate requires a more comprehensive study of glycoscience, particularly in the spatial distribution and the relationship with other related molecules which are rarely investigated. Recently, fluorescent imaging has been implemented to visualize the glycosylation process and identify the carbohydrate functions in cells or organisms, ${ }^{27,28}$ but the detailed distribution of carbohydrates or interactions of glycoconjugates cannot be acquired due to the limitation of imaging resolution. Fortunately, super-resolution fluorescence imaging with rapid development is a promising valuable tool in the carbohydrate study of cells, because of its predominant resolution at the nanoscale level. ${ }^{29,30}$

In this review, we mainly introduce the principle of super-resolution imaging, the fluorescent probes available for super-resolution imaging, some common labeling methods for carbohydrates, and the applications of super-resolution imaging in the study of glycoscience.

\section{The Principle of Super-Resolution Imaging}

With overcoming the diffraction barrier and significantly improving spatial resolution in all three dimensions over conventional light microscopy, superresolution fluorescence microscopy is able to elucidate biological processes at the molecular scale through observing previously unresolved details of cellular 
structure. It includes techniques with spatially patterned excitation, i.e., stimulated emission depletion (STED) microscopy ${ }^{31,32}$ reversible saturable optically linear fluorescence transitions (RESOLFTs) ${ }^{33}$ and saturated structured-illumination microscopy (SSIM), ${ }^{34}$ as well as techniques termed as single-molecule localization microscopy (SMLM), i.e., (direct) stochastic optical reconstruction microscopy ((d) STORM),$^{35,36}$ (fluorescence) photoactivated localization microscopy ((f)PALM). ${ }^{37,38}$

Here, we mainly introduce the imaging principle of SMLM. Generally, a typical biological sample labeled with extremely high density of fluorophores is difficultly resolved by the single molecule localization approach. However, with an ability to switch between a nonfluorescent and a fluorescent state by exposure to light, photoswitchable fluorescent probes positioned in close proximity can be activated at a quite low density at different time points by a weak activation laser illumination, as a result, only a sparse subset of molecules in a given region is imaged and localized with high precision. After these fluorescent molecules are deactivated, a new subset is activated and then localized. This cycle of activation, localization, and deactivation is repeated to acquire a sufficient number of localizations, and a high resolution image is reconstructed from numerous localizations. Therefore, based on the imaging principle, the resolution of final image is determined by the precision of localization and the localization density, rather than limited by diffraction.

\section{Fluorescent Probes Suitable for Super-Resolution Imaging}

Fluorescent probes available for super-resolution imaging can be switched from a nonfluorescent "off" state to a fluorescent "on" state by irradiation with light, and then back again to the "off" state. They normally include photoswitchable fluorescent proteins (psFPs) in (F)PALM, ${ }^{39}$ standard organic fluorophores in dSTORM, ${ }^{40,41}$ pairs of organic fluorophores-combinations of activator and reporter fluorophores in STORM, ${ }^{35,42}$ and other emerging small molecule labeling probes. ${ }^{43-46}$

\subsection{Photoswitchable fluorescent proteins}

Most psFPs in (F)PALM are synthesized in their "off" state and switched "on" by low doses of near-
UV light $(\sim 405 \mathrm{~nm})$. Then, they emit a burst of photons and are switched "off" again through photobleaching. The common psFPs include Dronpa, Padron, EosFP, tdEos, mEos2, PAmCherry1, and so on. Their detailed properties have been comprehensively demonstrated in some reviews ${ }^{39,47}$ Overall, fluorescent proteins are much smaller (a barrel with dimensions $2.4 \mathrm{~nm} \times 4.2 \mathrm{~nm}$ ) than fluorophorelinked antibodies (the size of an IgG antibody is $\sim 10$ $\mathrm{nm}$ ) which are typically used in dSTORM and STORM imaging. ${ }^{48}$ Besides, psFPs are genetically fused to target proteins and endogenously expressed in cells and organelles. ${ }^{49}$ Therefore, their outstanding advantages are imaging in live cells and achieving single molecule trajectory. ${ }^{50,51}$ However, the problem of maturation needs more cautions, and endogenous protein tagging should demonstrate the viability of wild type cells. Additionally, compared with standard organic fluorophores, lower photostability and brightness of psFPs as used in PALM will produce a lower resolution image.

\subsection{Photoswitchable fluorescent dyes}

Small organic fluorophores can be switched between a bright "on" and a long-lived dark "off" state upon irradiation with different wavelengths in the absence $^{52}$ or presence of a second activator fluorophore $^{53}$ when imaged in buffers containing oxygen scavengers and/or reducing agents. Upon excitation, the fluorophore is cycled between the singlet ground state and the first excited state with emitting fluorescence photons. In intersystem crossing, the triplet state is quenched by molecular oxygen or appropriate reducing agents, consequently, singlet oxygen repopulates the singlet ground state or the fluorophore radical anion is generated. Generally, the fluorophore is expected to cycle several times between singlet ground and excited state to generate as many detectable photons as possible before the triplet state is reduced and a very stable "off" state is generated (with lifetime of seconds to minutes). A wide variety of conventional fluorescent dyes can be used as reversible photoswitches in the presence of imaging buffer, including cyanine dyes (i.e., Cy5, Cy5.5, Cy7 or Alexa Fluor 647, 680, and 700), rhodamine dyes (Alexa Fluor 488, 532, Cy3), oxazine and thiazine dyes (i.e., methylene blue, ATTO 655, and ATTO 680). More details of standard organic fluorophores can refer to the literatures. ${ }^{41,54}$ With advantages of high photostability 


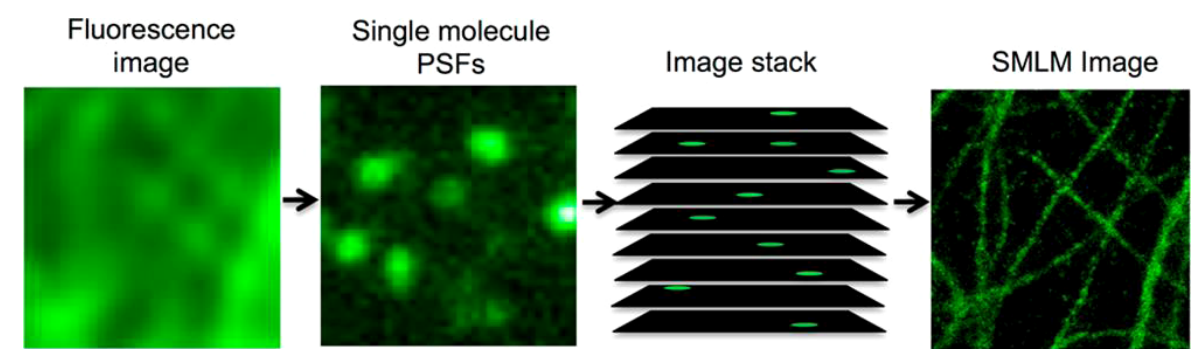

Fig. 1. Imaging principle of SMLM. The diffraction-limited epi-fluorescence image where all fluorophores emit photons (panel 1). For SMLM, a majority of fluorophores are switched to a dark state, allowing subsets of fluorophores to be imaged without spatial overlap and to be localized at high precision (panel 2). The activation and imaging processes are iterated to accumulate thousands of frames of single-molecule emissions (panel 3) and a super-resolution image is reconstructed from numerous localized emissions (panel 4). Adapted with permission from Ref. 29.

and high photon yield, photoswitchable fluorescent dyes are efficient probes for super-resolution imaging, especially for (d)STORM imaging. However, most fluorescent dyes are involved in redox chemical processes during imaging process; as a result, they are difficult to be available for live-cell imaging. Thus, more novel fluorophores or combined labeling strategies which enable live-cell localization should be developed. For example, G. Lukinavičius et al. synthesized a biocompatible near-infrared silicon-rhodamine probe suitable for live-cell super-resolution imaging, with being coupled specifically to proteins using different labeling techniques. ${ }^{55} \mathrm{R}$. Wieneke et al. developed a small labeling pair (SLAP) based on a synthetic smallmolecule recognition unit (Ni-trisNTA) and the genetically encoded minimal protein $\mathrm{His}_{6-10}$-tag to label target molecules with high density to fulfill the necessary sampling criteria for SMLM. ${ }^{56}$ As seen in Fig. 2, with conjugating Alexa 647 to Ni-trisNTA allowing to make benefits of organic dyes (small size, superior quantum yield, photostability), they performed dSTORM imaging to visualize the His ${ }_{10^{-}}$ tagged proteins (actin and laminA) via SLAP.

\subsection{Emerging small molecular labeling methods}

In recent years, other effective labeling strategies have been developed to label different types of biomolecules. Especially, small probes have been applied to label the targets with high density for accurate super-resolution imaging. Ries and colleagues firstly demonstrated that small nanobodies $(13 \mathrm{kDa}, \sim 3 \mathrm{~nm})$ contributed to improve the precision of molecular localization in GFP-based
PALM microscopy. ${ }^{57}$ Single-chain antibody fragments (nanobodies) against tubulin was engineered to achieve super-resolution imaging of microtubules with a decreased apparent diameter. ${ }^{58}$ In parallel, aptamers (short single stranded oligonucleotides $(\sim 15 \mathrm{kDa}, \sim 4 \mathrm{~nm})$, as small as nanobodies, can also benefit the STED imaging when compared with antibody staining, ${ }^{59}$ as seen in Fig. 3. Moreover, bio-orthogonal click chemistry, by incorporating a unique chemical group (i.e., azide or alkyne) into a target molecule (i.e., nucleotide, nucleoside, amino acid, monosaccharide, or fatty acid) through the cell's own biosynthesis machinery, has been utilized in super-resolution imaging with improving biocompatibility and labeling condition. Zessin and colleagues are the first to successfully combine 5-Ethynyl-20-deoxyuridine (EdU) labeling and dSTORM technology for super-resolution imaging of DNA. ${ }^{60}$ Although these emerging probes can improve the imaging quality of SMLM in some aspects, the category of biomolecules labeled by these strategies is limited and impedes their wide applications in super-resolution imaging.

The ideal labeling probes in super-resolution imaging are small, bright, and photostable fluorophores which can preferably and directly attach to the molecule of interest. Therefore, more effective fluorescent probes need to be developed or improved to benefit super-resolution imaging.

\section{The Strategies of Labeling Carbohydrates}

Because of the low affinity to carbohydrates which principally depend on numerous hydroxyl 


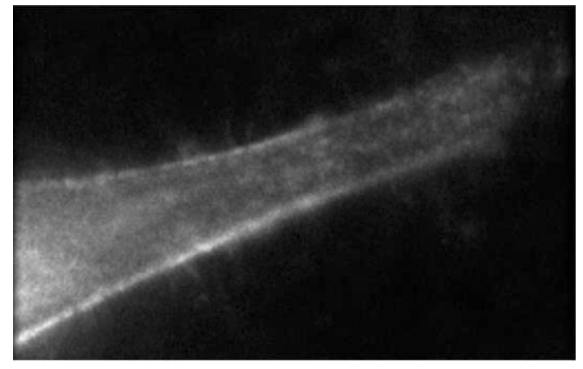

(a)

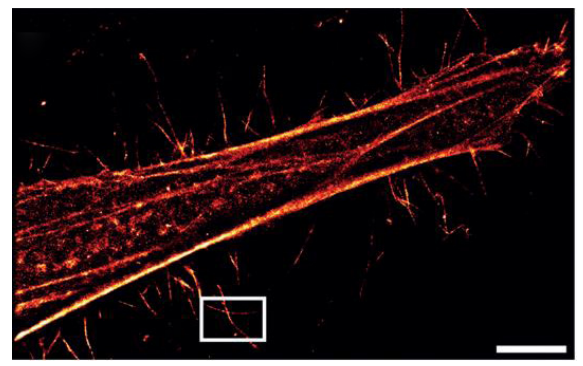

(b)

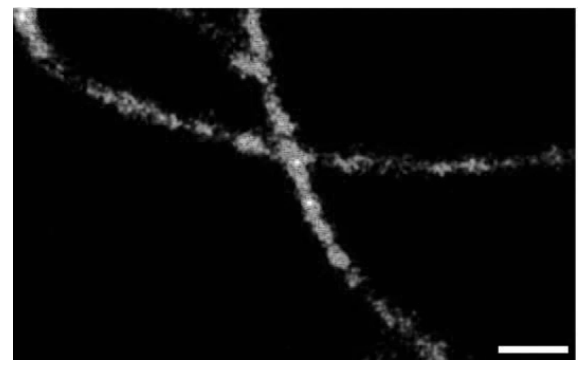

(c)

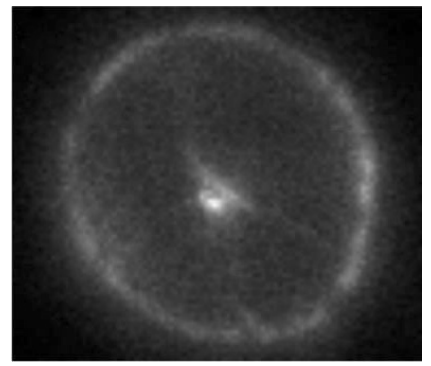

(d)

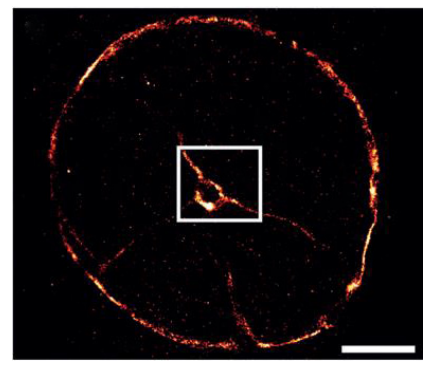

(e)

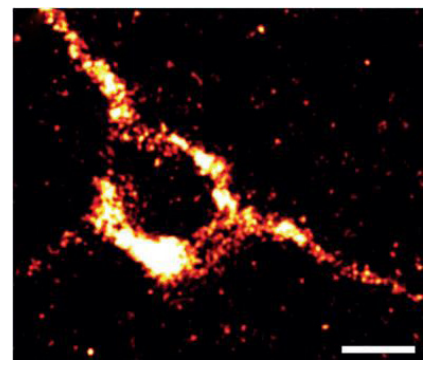

(f)

Fig. 2. Single-molecule super-resolution imaging of His 10 -tagged proteins labeled with Ni-tris-NTA ${ }^{\text {AlexaFluor647 }}$. Compared with conventional fluorescence images (a) and (d), reconstructed dSTORM images of actin (c) and laminA (d) exhibit higher optical resolution. Magnifications of boxed areas illustrate the high-density labeling and superior resolution obtained by using SLAP. Scale bars are $5 \mu \mathrm{m}$ in (b), $2.5 \mu \mathrm{m}$ in (e), and $0.5 \mu \mathrm{m}$ in (c) and (f). Adapted with permission from Ref. 56 .

groups, as well as the large diversity and complexity of carbohydrate structures, it is more difficult to develop the recognition molecules for binding carbohydrates than proteins. So far, carbohydraterecognition molecules mainly include natural lectins, synthetic lectins from organic chemistry, small biological molecules (aptamers and peptides) and engineering of neo-lectins from glycosidases and lectins. Here, we focus on the natural lectins, aptamers and peptides that are most potential to be utilized to label carbohydrates in super-resolution imaging. Besides, the bio-orthogonal metabolic labeling of carbohydrates is increasingly improved for imaging of some types of carbohydrates.

\subsection{Natural lectins for recognizing carbohydrates}

Generally, lectins are thought to be superior to anticarbohydrate antibodies. The most important reason is the limited specificity of anti-carbohydrates antibodies. Considering the structural diversity of carbohydrates, we need to prepare a set of antibodies (the number of antibody $>10,000$ ) to identify all probable carbohydrates. On the other hand, the precise affinity of anti-carbohydrate antibody is not determined systematically. Thus, specific antiantibodies are not suitable for comprehensive carbohydrate analysis. However, a repertoire of lectins can work for this purpose with their board 

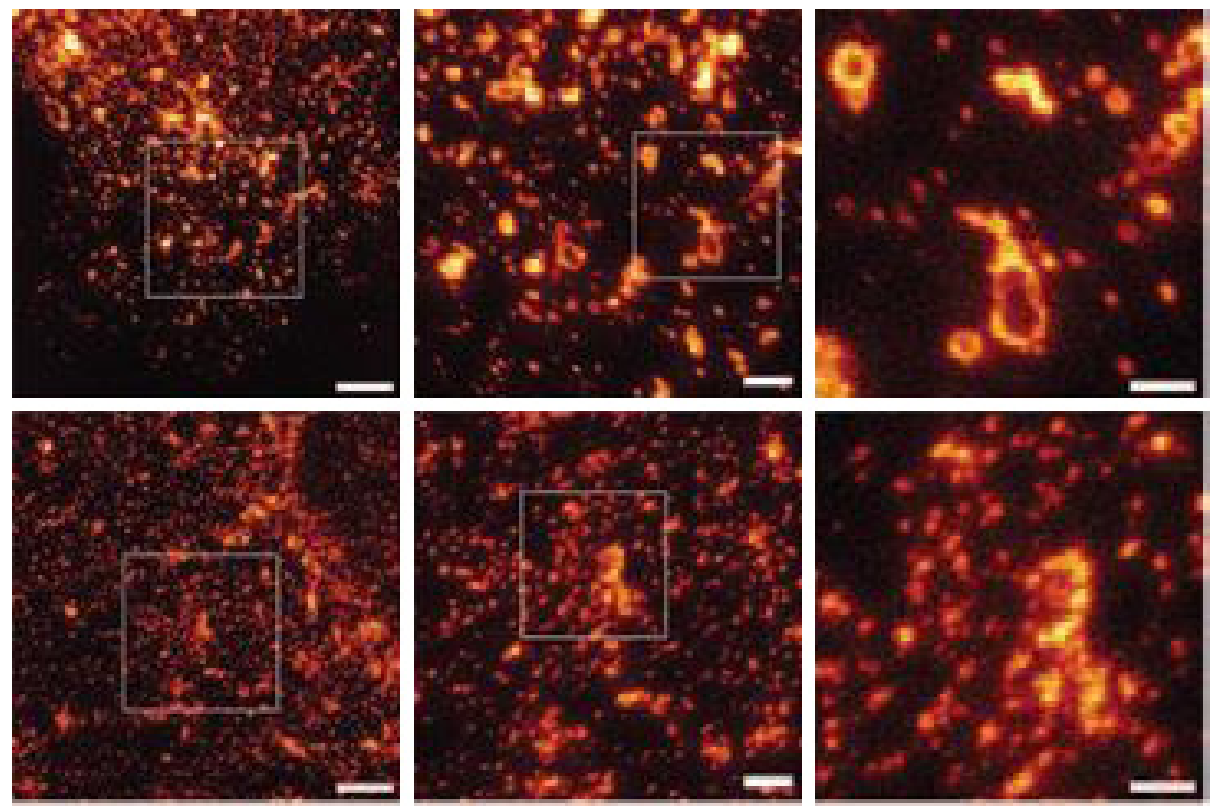

Fig. 3. STED images of cells stained with the transferrin receptor (TfnR) aptamer c2 (top) or with a monoclonal TfnR antibody (bottom). Scale bars are $3 \mu \mathrm{m}$ in left, $1 \mu \mathrm{m}$ in middle, and $500 \mathrm{~nm}$ in right, respectively. Adapted with permission from Ref. 59.

specificity from their ubiquity and great variety. Overall, because of commercial availability, stability, diverse specificities, and economy, natural lectins are the most widely used in study of glycoscience among diverse bio-recognition molecules of carbohydrates.

Lectins can specifically recognize free carbohydrates and those presenting on glycoconjugates without altering the structure of carbohydrates or conjugated molecules. As a consequence, lectins play a significant role in deciphering the glyco-code. Routinely, lectins are exploited to label specific carbohydrate-epitopes on cell surfaces, characterize carbohydrate structures, and purify glycoproteins in a variety of analysis technologies (i.e., flow cytometry, histochemical staining, western blot analysis, lectin-affinity chromatography). Recently, lectin-based glyco-biosensors and lectin microarrays have been widely applied in biotechnology, diagnostic, and therapeutic researches, with the ability to directly analyze even crude samples containing glycoproteins and reveal the differential profiles of carbohydrates on various cells. ${ }^{61}$ Additionally, lectins are linked with fluorescent molecules to form specific carbohydrate probes used for locating the cell surface carbohydrates via conventional fluorescence microscopy. For example, Lescar et al. utilized biotin or Cy5-linked Helix pomatia agglutinin (HPA) for locating GalNAc on various cancer cell lines, with negative control experiments showing the labeling is dependent upon the presence of carbohydrate epitopes, as shown in Fig. $4 .{ }^{62}$

\subsection{Small biological molecules: Aptamers and peptides}

Higher affinity and specificity of aptamers make them as attractive alternatives to lectins or antibodies in detection probes of carbohydrates. Generally, systemic evolution of ligands by exponential enrichment (SELEX), along with repetitive cycles of selection and amplification, is a powerful selection method for various aptamers of the specific cell surface biomarkers. ${ }^{63}$ It utilizes a random library of oligonucleotide candidates for the in vitro selection of ssDNA and RNA molecules with best binding specificity to an affinity immobilized target, then these selected ssDNA and RNA are retained and amplified to be selected again in a new cycle, as seen in Fig. 5. So far, several aptamers have been reported using various types of carbohydrate recognition, including monosaccharides (D-galactose, D-glucose, and D-mannose), ${ }^{64}$ disaccharides (cellobiose), ${ }^{65}$ oligosaccharides (sialylactose, sialyl Lewis $\left.\mathrm{X}\left(\mathrm{sLe}^{X}\right)\right),{ }^{66,67}$ polysaccharides (cellulose, chitin, and curdlan), ${ }^{68,69}$ and carbohydrates from glycoproteins (fibrinogen). ${ }^{70}$ The detailed and comprehensive introduction can refer to the related 

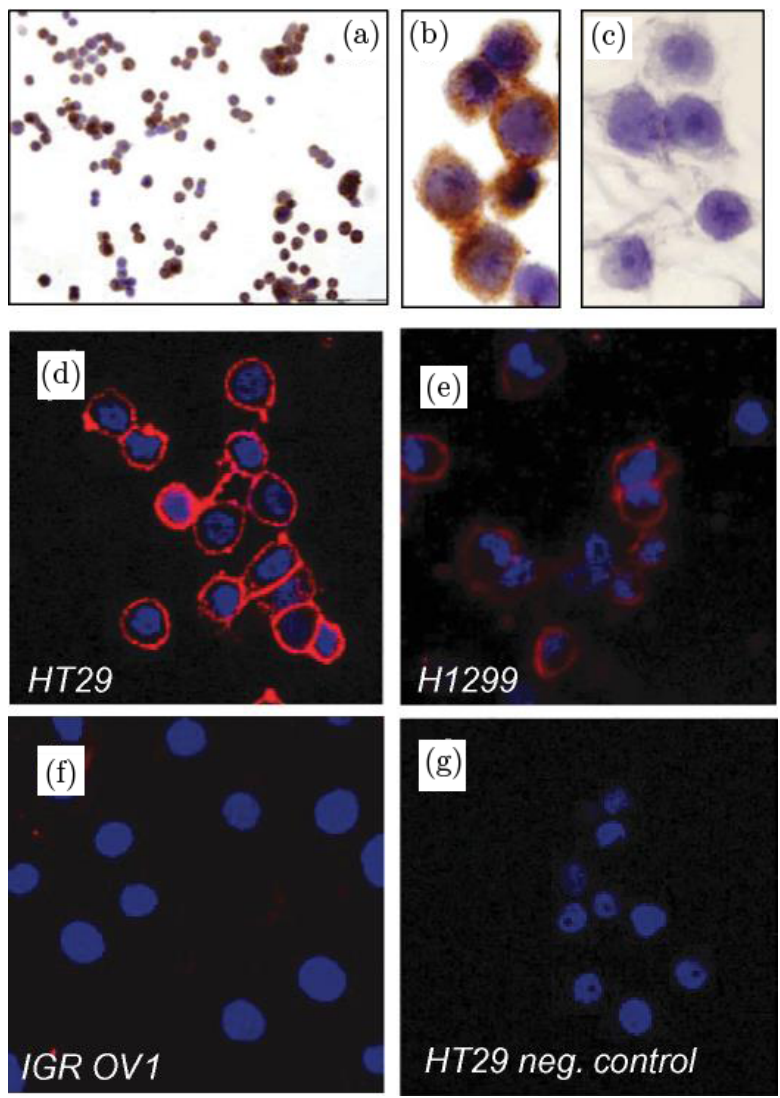

Fig. 4. Specific labeling of various tumor cells by HPA (the GalNAc-specific lectin from Helix pomatia). (a) Biotin-HPA labeling of HT29 cancer colon cells, Scale bar is $100 \mu \mathrm{m}$. (b) Enlarged image of the labeled cells. (c) Negative control in the presence of $100 \mathrm{mM}$ GalNAc. Cy5-HPA labeling of (d) HT29 colon cancer cell lines, and (e) human nonsmall cell lung cancer H1299. (f) Negative labeling of IGROV1 ovarian cancer cell lines and (g) control experiment in presence of $100 \mathrm{mM}$ GalNAc. (d)-(g): nuclei are blue stained with $5 \mu \mathrm{M}$ Hoechst 33342). Adapted with permission from Ref. 62.

review. ${ }^{71}$ However, most carbohydrate-recognition aptamers were developed to identify or block specific carbohydrates on cell surfaces. It is rare to utilize these aptamers for locating carbohydrates. Therefore, there is a great development room for imaging probes of carbohydrates, particularly in super-resolution imaging.

Carbohydrate-recognition peptides contain natural ones and engineered ones. The natural ones are commonly derived from natural lectins or selected via biotechnology. Odorranalectin only composed of 17 residues is the smallest natural peptide with lectin activity and specific for fucose. ${ }^{72}$ Besides, carbohydrate-binding peptides can be obtained by proteinase digestion of legume lectins. For example, $\alpha$-GalNAc-specific peptide is from cytisus scoparius seed lectin, ${ }^{73}$ fucose-specific one is from ulex europeus agglutinin I (UEA-I) and lotus tetranogologbus agglutinin, ${ }^{74}$ GlcNAc-specific one is from UEA-II, ${ }^{74}$ and Gal-specific one is from bauhinia purpurea lectin. ${ }^{75}$ The engineered peptides can be designed and synthesized from the crystal structure of toxins or lectins, such as, the peptide from pertussis toxin can bind to sialylated conjugates,${ }^{76}$ the one from E-selectin was identified to bind to $\mathrm{SLe}^{\mathrm{X}}, 77$, and the one was designed to mimic the binding sites of siglec family. ${ }^{78}$ Additionally, novel carbohydrate-binding peptides were acquired by phage-display technology (a phage display approach followed by biopanning), including the peptide against $\mathrm{T}$ antigen $(\mathrm{Gal} \beta 1-3 \mathrm{GalNAc}),{ }^{79}$ the ones against sialylated, ${ }^{80}$ and sulfated Lewis antigens. ${ }^{81}$ With the development of high-throughput assays and cost-efficient chemical synthesis of peptides, random sequence peptide arrays containing 1000 peptides were used with luminescent glyconanoparticles to identify two sets of peptides that can bind to $3^{\prime}$-sialylactose and 6 -sialylactose. ${ }^{82}$ Besides, some modified peptides with higher affinity and specificity have been developed to better 


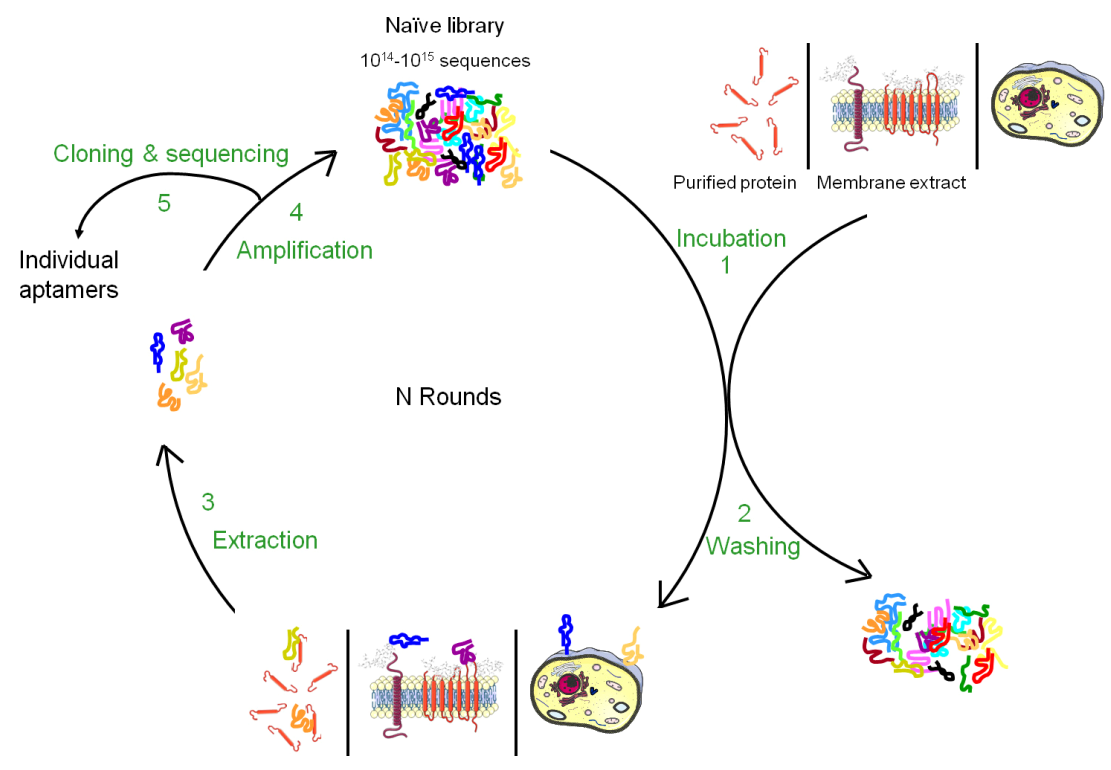

Fig. 5. The general principle of the SELEX procedure for a cell surface biomarker. (1) Oligonucleotide-based library is incubated with a target (purified cell surface biomarker, membrane extract or whole living cell or organism). (2) Using different partitioning methods (i.e., affinity chromatography, filtration, centrifugation) to remove unbound sequences. (3) Bound sequences are eluted (i.e., urea, EDTA, competition with a ligand). (4) Amplification by PCR (or RT-PCR and in vitro transcription in the case of RNA libraries). The selected pool then enters a new cycle of selection, repeating rounds of selection to evolve the population of the sequences. (5) Finally, sequences are cloned and sequenced to identify the aptamers. Adapted with permission from Ref. 63.

detect carbohydrates. For example, $\mathrm{Xu}$ et al. functionalized peptides with boronic acid as fluorescent sensors (BPFSs) for in situ recognition and differentiation of cancer-associated carbohydrates, as well as targeted imaging of cancer cells. ${ }^{27}$ Similar to aptamers, although numerous identified carbohydrate-binding peptides have been applied to detect their specific carbohydrates in cells or other organisms, they are rarely used as probes in the imaging of carbohydrates. In the near future, it is promising to combine these aptamers or peptides with photoswitchable fluorescent probes to implement superresolution imaging of carbohydrates, which will make a great progress in the study of glycoscience.

\subsection{Bio-orthogonal metabolic labeling for carbohydrates}

In brief, bio-orthogonal chemistry refers to chemical reactions that can occur inside of living systems without interaction or interference with biological systems. Using bio-orthogonal chemistry to label carbohydrates typically includes two steps, ${ }^{83}$ as seen from Fig. 6. Firstly, cellular carbohydrate substrates modified with a bio-orthogonal functional group (chemical reporter) are introduced to cells and incorporated into newly synthesized carbohydrates. Secondly, imaging probe containing a complementary functional group is added to label the substrate bio-orthogonally. To successfully label carbohydrates in living systems, some requirements need to be fulfilled: (1) both the reporter group and the probe must be small and biologically inert; (2) the functional group needs no cellular toxicity but high selectivity; (3) the reaction should occur in physiological environment with very fast kinetics, particularly at low concentrations. A series of developments in reporter reactions has made great progress in the imaging of carbohydrates, such as, the azide can be detected by reacting with phosphines via the staudinger ligation, ${ }^{84}$ 1,3-dipolar cycloaddition between the azide and linear alkynes under catalysis of $\mathrm{Cu}$ (abbreviated CuAAC), a variety of interactions of cyclooctynes and azides via $\mathrm{Cu}$-free click chemistry, the reaction of pyradazine or dihydropyrdazine synthesized from tetrazines with various unsaturated compounds via inverse-demand Diels-Alder reaction, and the isonitrile based click chemistry. So far, many carbohydrates have been labeled with bio-orthogonal chemical reporters, such as, sialic acid (Sia) was metabolically labeled with its biosynthetic 


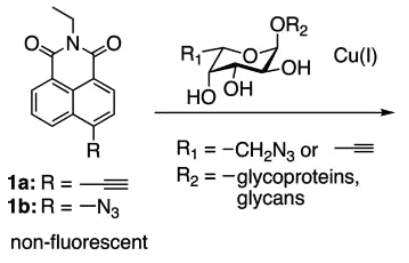

(a)

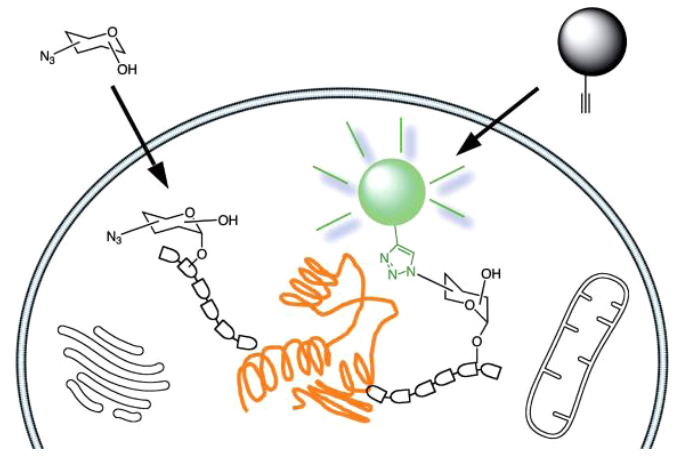

(b)

Fig. 6. General bio-orthogonal metabolic labeling strategy for carbohydrates. (a) Probe structures including an azide or alkyne at a position of the ring allows a fluorogenic ligation with 6-modified fucose analogs. Probes react with the azido_alkynyl group of fucosides through $\mathrm{Cu}(\mathrm{I})$-catalyzed $[3+2]$ cycloaddition and generate the fluorescence adduct. (b) Strategy for specific fluorescent labeling of fucosylated glycans in cells. The production of fluorescently labeled glycoproteins bearing modified fucose (azido fucose shown) is caused by covalently modifying the target glycan with probes 1a or 1b. Adapted with permission from Ref. 83 .

precursor N-azudoacetylmannisamine (Man$\mathrm{NAz}$ ), ${ }^{84,85}$ N-azudoacetylneuraminic acid (SiaNAz), ${ }^{85,86}$ and alkynyl ManNAc. ${ }^{87}$ The core GalNAc was labeled by feeding cells or animals with per-O-acetylated $\mathrm{N}$-azidoacetylgalactosamine $\left(\mathrm{Ac}_{4} \mathrm{GalNAz}\right){ }^{28,88}$ The metabolic labeling of $\mathrm{O}-$ GlcNAc was achieved by using per-O-acetylated Nazidoacetylgalactosamine $\left(\mathrm{Ac}_{4} \mathrm{GlcNAz}\right)$ or $\left(\mathrm{Ac}_{4} \mathrm{GalNAz}\right) .{ }^{89}$ Fucose was labeled by using perO-acetylated 6-azidofucose $(6 \mathrm{AzFuc})^{90}$ or per-Oacetylated 6-alkynylfucose (alkynyl fucose). ${ }^{87} \mathrm{In}$ addition to the carbohydrates in cells, membraneassociate carbohydrates in live organism (developing zebrafish) were firstly imaged by Laughlin et al. via the stain promoted cycloadditon of azide with a cyclooctyne reagent bearing a gem-difluoro group adjacent to the alkyne (termed "DIFO" regents). ${ }^{91}$ Recently, Robinson and co-workers used cyclooctyne-aptamer conjugates to label specific protein glycoforms in live cells, and showed their labeling method can be applied to detect protein sialoforms in mass spectrometry, western blotting, and flow cytometry. Unfortunately, due to poor signal-tonoise after washing, their strategy cannot be applied to fluorescent microscopy. ${ }^{92}$ Overall, there are some challenges in carbohydrates imaging with bioorthogonal chemical reporters, including (1) some unnatural carbohydrate substrates may poorly incorporate into the cells, (2) some designed carbohydrate substrates cannot specifically convert to the target carbohydrates, (3) some reported reactions lack high specificity and have high background fluorescence. Therefore, more effort needs to be devoted in the development of bio-orthogonal reactions, especially those available for super-resolution imaging.

\section{Applications of Super-Resolution Imaging Technology in Spatial Investigation of Carbohydrates}

Although super-resolution imaging technology has been widely applied in biological field, there are only few studies of carbohydrates via super-resolution strategy reported, which may result from the lack of suitable carbohydrate-binding probes available for super-resolution imaging. Letschert et al. combined super-resolution imaging and metabolic labeling to visualize membrane-associated glycoproteins with subdiffraction resolution. ${ }^{93}$ They fed cells with $\mathrm{Ac}_{4} \mathrm{ManNAz}$ and $\mathrm{Ac}_{4} \mathrm{GalNAz}$ (analogues of biosynthetic precursors ManNAc and GalNAc) which were incorporated into cell surface carbohydrates upon cell permeation and deacetylation, then metabolically labeled Sia and mucintype O-linked glycans by reacting alkyne-bearing Alexa 647 with catalysis of $\mathrm{Cu}(\mathrm{I})$. Similarly, they labeled the O-GlcNAc modified plasma membrane proteins by using a GlcNAc analogue $\left(\mathrm{Ac}_{4} \mathrm{Glc}-\right.$ $\mathrm{NAz}$ ). By dSTORM imaging, they found these examined carbohydrates homogeneously distributed over the entire basal plasma membranes of U2OS and SK-N-MC cells with high localization densities 


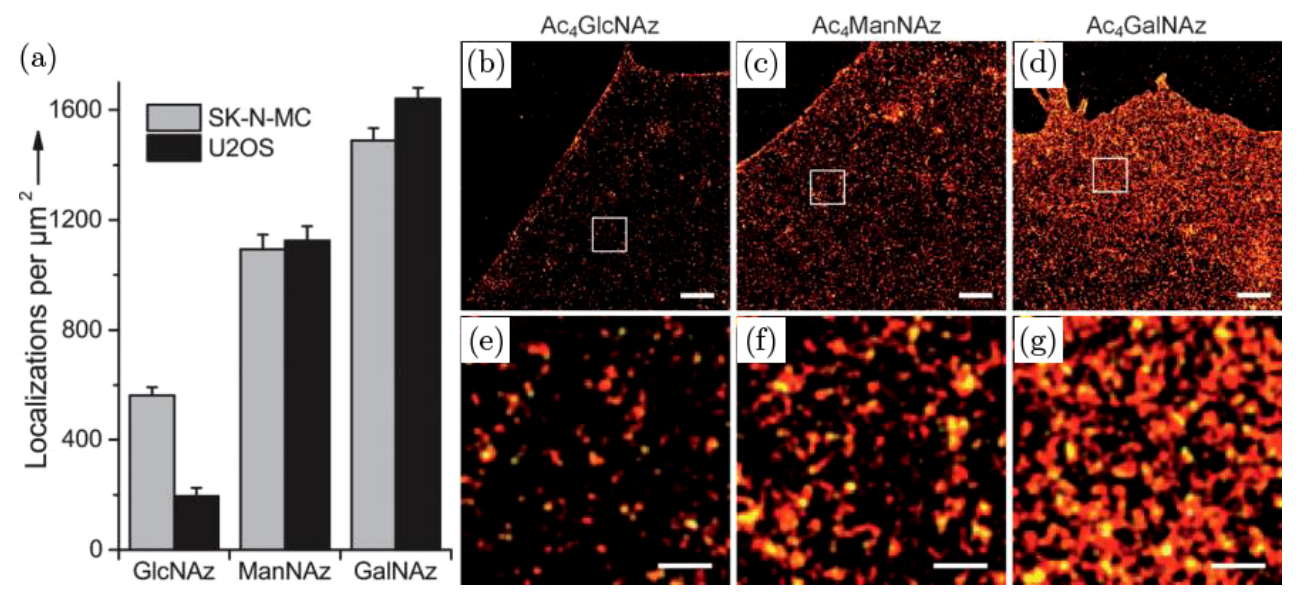

Fig. 7. Super-resolution imaging and analysis of cell-surface glycoproteins containing Sia or GlcNAc. Combination of click chemistry and dSTORM imaging to visualize and analyze the carbohydrates on SK-N-MC neuroblastoma cells and U2OS cells fed with one of the reactive azido sugars ( $\mathrm{Ac}_{4} \mathrm{GlcNAz}, \mathrm{Ac}_{4} \mathrm{ManNAz}$, or $\mathrm{Ac}_{4} \mathrm{GalNAz}$ ). (a) Localization density of membrane-associated carbohydrates labeled with Alexa $647(20 \mu \mathrm{M})$. Error bars are the standard error of the mean of 12-32 imaged cells. (b)-(d), dSTORM images of glycoconjugates in the basal membrane of SK-N-MC neuroblastoma cells. (e)-(g), Enlarged images of white boxed regions. Scale bars are $1 \mu \mathrm{m}(\mathrm{b})-(\mathrm{d})$ and $200 \mathrm{~nm}(\mathrm{e})-(\mathrm{g})$. Adapted with permission from Ref. 93.

(Fig. 7). Their work confirmed click chemistry can be applied into super-resolution imaging technology to study the distribution of carbohydrates on cell membranes.

Jiang and co-workers metabolically labeled Nlinked Sia and GalNAc with bio-orthogonal copper (I)-catalyzed azide-alkyne cycloaddition, then conjugated these azide- or alkyne-tagged carbohydrates to the controllable bleaching of fluorescent probes (Alexa 647, Alexa 488) and finally carried out dynamic single-molecule tracking and super-resolution imaging of Sia and GalNAc. ${ }^{94}$ In detail, they cultured PyMT (mouse mammary carcinoma cell line) and Met-1 (the highly metastatic variant) in medium supplemented with either $\mathrm{Ac}_{4} \mathrm{GalNAz}$ (which is metabolized and installed on mucin-type O-linked glycoproteins by the ploypeptide $\mathrm{N}$-acetyl- $\alpha$-galactosaminyl transferase), or $\mathrm{Ac}_{4} \mathrm{ManNAl}$ (peracetylated $\mathrm{N}$-(4-pentynoyl) mannosamine). Then, cell-surface mucin-type O-carbohydrates and Sia were labeled by reacting with either an alkyne or azide-linked Alexa Flour under the catalysis of biocompatible BTTPS $/ \mathrm{Cu}(\mathrm{I})$. With reduction of the initially high spatial density, they dynamically tracked hundreds of diffusing single molecules for hundreds of frames and analyzed the individual trajectories of the cell surface carbohydrates. Additionally, with the similar labeling scheme, they performed STORM imaging to visualize Sia in Hela cells through labeling with $\mathrm{Ac}_{4} \mathrm{ManNAl}$ and conjugating with Alexa 647-azide (Fig. 8). Their work demonstrates that this metabolically labeling method for carbohydrate can provide a carbohydrate-recognition probe available for super-resolution imaging of the carbohydrates in live cell membranes.

This metabolic labeling approach coupled with bio-orthogonal chemistry has been successfully applied to super-resolution imaging of carbohydrates. However, there are some challenges that need to be faced. Based on the protocol of this labeling method, we know that the labeled carbohydrates are not the natural ones, and thus lead to a different result from the native ones. Owing to the limited types of analogues of biosynthetic precursors of specific carbohydrate, the category of carbohydrates labeled by this methods is a minority. Besides, some synthesized carbohydrate precursors are poorly incorporated into the cell surface carbohydrates,${ }^{89,95,96}$ which may lead to an incomplete localization of carbohydrates. Moreover, most of chemical ligation strategies are probably unsuitable for super-resolution imaging, especially for living cell imaging, due to their slow reactivities, high background fluorescence, or toxicity to cells. For example, cyclooctyne-aptamer conjugates were developed by Robinson et al. to label specific glycoforms in mass spectrometry, western blotting, and flow cytometry, but except for fluorescent microscopy owing to poor signal-to-noise after washing. ${ }^{92}$ 


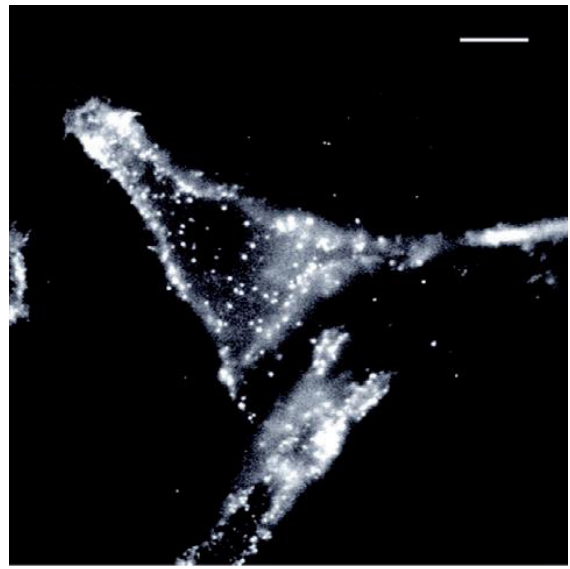

(a)

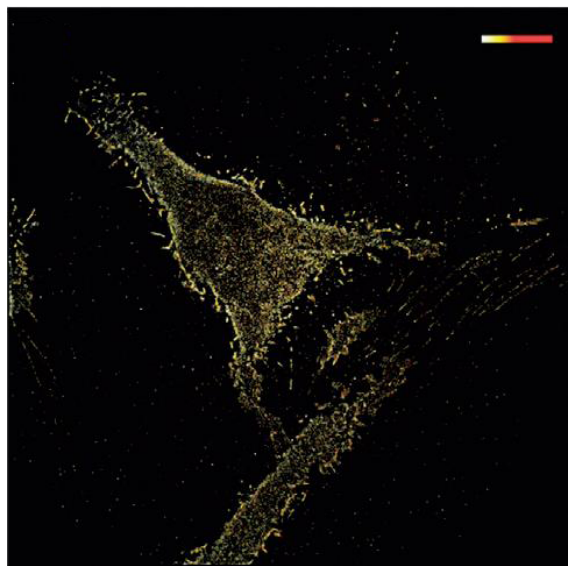

(b)

Fig. 8. STORM imaging of Sia in HeLa cells by metabolically labeling with $\mathrm{Ac}_{4} \mathrm{ManNAl}$ and interaction with Alexa Fluor 647 azide. (a) Single STORM image (100th frame) from a sequence. (b) The STORM image reconstructed from 480 consecutive frames. The color bar represents the integrated fluorescent intensity of each molecule. Scale bars: $10 \mu$ m. Adapted with permission from Ref. 94.

Chen et al. performed dSTORM imaging to visualize the distribution pattern of cell surface carbohydrates on Vero cells, through labeling GlcNAc with Alexa 647-linked WGA. ${ }^{97}$ They revealed the differential morphologies of GlcNAc on the fixed Vero apical and basal membranes at the nanoscale resolution (Fig. 9). That is, GlcNAcs were mainly distributed into larger clusters on Vero apical membranes at a higher cluster density, compared with those on Vero basal membranes. Additionally, GlcNAc and lipid raft were reveal to be mostly colocalizated by preforming dual-color dSTORM

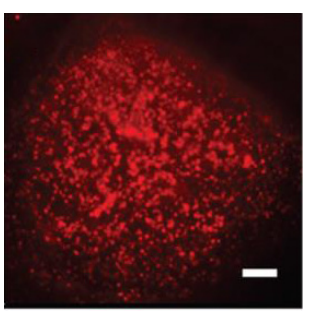

(a)

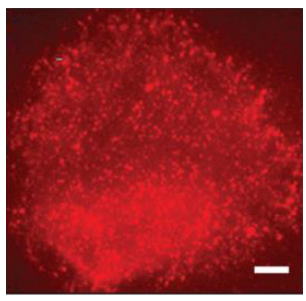

(e)

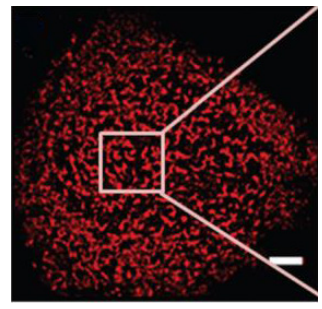

(b)

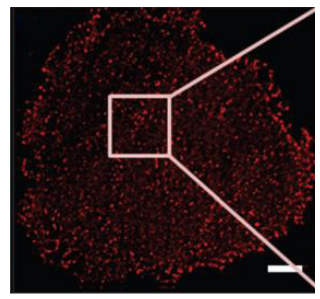

(f)

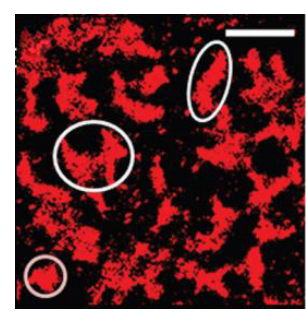

(c)

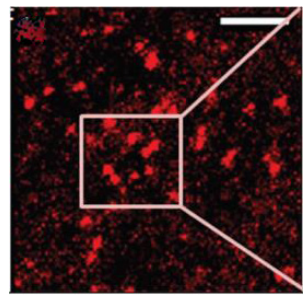

(g)

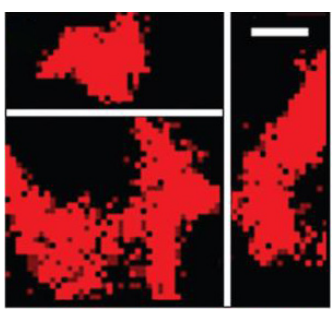

(d)

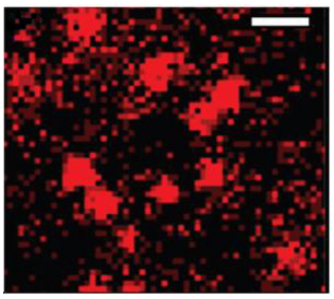

(h)

Fig. 9. Super-resolution images of GlcNAcs on the Vero apical and basal membranes by labeling with Alexa 647-linked WGA. (a) and (e) the blurry conventional TIRF images of GlcNAcs on the Vero apical (a) and basal (e) membranes. [(b) and (f)] The corresponding dSTORM images present the refined distribution of GlcNAcs on the cellular membranes with nanoscale resolution. [(c) and (g)] The enlarged images of the boxed regions in (b) and (f) show the detailed distribution differences of GlcNAcs. [(d) and (h)] The further enlarged images of three representative clusters of GlcNAcs on the apical surface and almost uniform and small clusters on the basal membrane. Scale bars are $5 \mu \mathrm{m}$ in (a), (b), (e), and (f), are $2 \mu \mathrm{m}$ in (c) and (g), and $200 \mathrm{~nm}$ in (d) and (h). Adapted with permission from Ref. 97. 


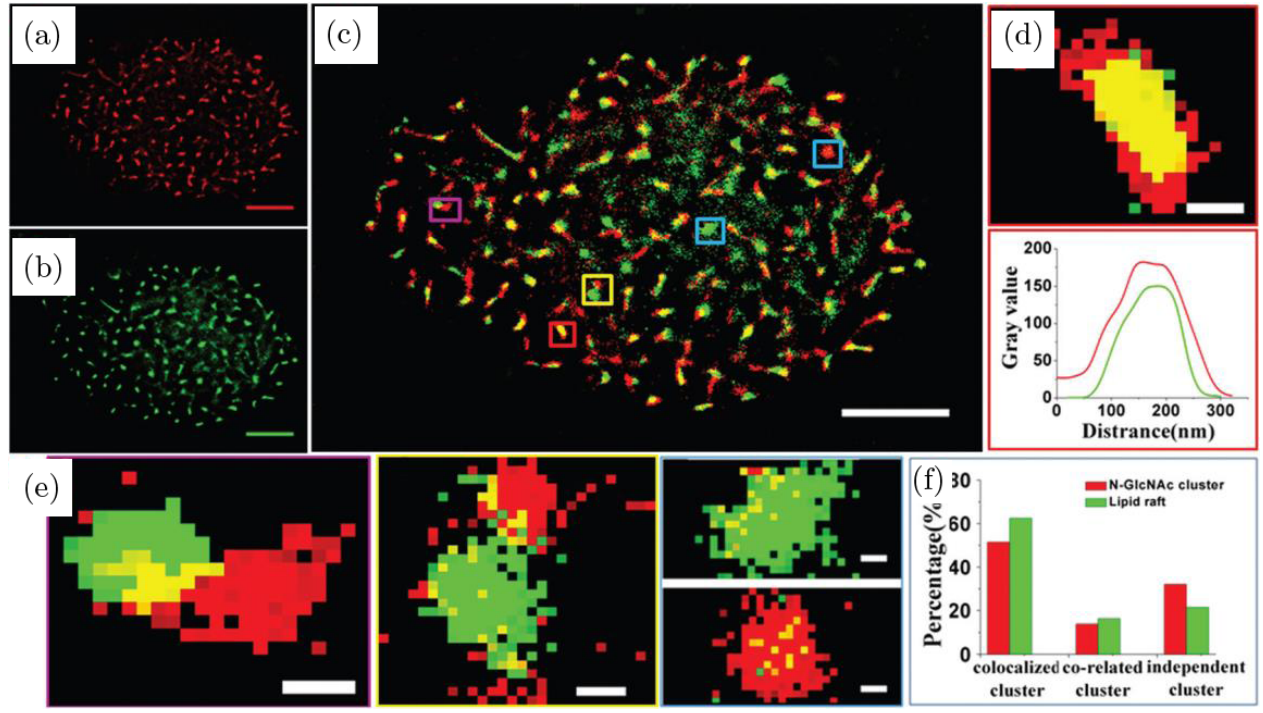

Fig. 10. Dual-color dSTORM imaging of GlcNAc clusters and lipid-raft domains on the Vero apical membranes. [(a) and (b)] dSTORM images of GlcNAcs and lipid rafts on the representative Vero apical surface, by being labeled with Alexa647-linked WGA and lipid raft marker, respectively. (c) Composite graph of (a) and (b), showing that two types of molecules are mostly co-localized. (d-up) Magnified image of the red box region in (c), displaying a typical co-localized relationship of two domains, which further illustrated by the corresponding intensity profiles of two domains (d-below). (e) Enlarged images of boxed regions in (c) classify three types of positional relationships: the co-localized domains (left), the related domains (middle), and the independent domains (right). (f) Histogram of three types of domains from 10 cells in three independent experiments. Scale bars are $5 \mu \mathrm{m}$ in (a) $-(\mathrm{c})$, and $200 \mathrm{~nm}$ in (d) and (e). Adapted with permission from Ref. 97.

imaging, suggesting GlcNAc clusters in combination of lipid raft were probably as functional domains (Fig. 10). Then, most GlcNAc clusters became smaller or even disappeared with treatment of methyl- $\beta$ cyclodextrin that can effectively extract cholesterol to disrupt lipid raft, which further confirms that the stable existence of most GlcNAc clusters was dependent on the intact lipid raft. Their work demonstrates that dSTORM imaging enables to localize carbohydrates in their biological environment on a molecular level, and a comprehensive view of the distributions of all carbohydrates on the plasma membrane will be realized with more efforts. Additionally, the localization relationships with other membrane molecules can also be revealed to provide a novel view about the structure of cell membranes.

About the different imaging results of carbohydrates, the main reason is thought to be different labeling methods, besides the specificity of cells. Metabolic labeling method used by Letschert et al. imaged the unnatural carbohydrates. However, through being labeled with Alexa Fluor-linked lectins, all of natural GlcNAcs on the cell membrane were almost detected supported by the experiment of concentration gradient.

\section{Challenges and Perspectives}

So far, there has been no general and accepted high performance labeling method for localizing carbohydrates via super-resolution fluorescence imaging. Metabolic labeling method is indeed a promising method, but with some of the above challenges. Various lectins combined with fluorescent probes possessing photoactivable or photoswitchable properties can be widely and flexibly applied in single or multi-color super-resolution imaging to study various classes of carbohydrates. But this method is limited to the fixed cells because of the potential cross-linking of lectins to carbohydrates. Some other probes (i.e., aptamer or peptide) have recently been developed to label carbohydrates, but this labeling method is rarely applied to super-resolution imaging of carbohydrates. Excitingly, some aptamers or peptides have been increasingly confirmed to be suitable for super-resolution imaging of some biomolecules (i.e., some proteins or DNA). Therefore, there is a great promise to develop novel fluorescent probes (i.e., aptamers or peptides specific to some carbohydrates) available for superresolution imaging. With more probes for carbohydrates being utilized in super-resolution imaging, 
a better and comprehensive understanding of carbohydrates at single molecule level will greatly promote the development of glycoscience.

\section{Acknowledgments}

This work was supported by NSFC (Grants 31330082, 21373200, 21525314), and the Instrument Developing project of the Chinese Academy of Sciences (Grant YZ201455).

\section{References}

1. A. Ishio, T. Sasamura, T. Ayukawa, J. Kuroda, H. O. Ishikawa, N. Aoyama, K. Matsumoto, T. Gushiken, T. Okajima, T. Yamakawa, "O-fucose monosaccharide of Drosophila Notch has a temperature-sensitive function and cooperates with O-glucose glycan in Notch transport and Notch signaling activation," J. Biol. Chem. 290, 505-519 (2015).

2. K. Kaszuba, M. Grzybek, A. Orłowski, R. Danne, T. Róg, K. Simons, Ü. Coskun, I. Vattulainen, "N-Glycosylation as determinant of epidermal growth factor receptor conformation in membranes," Proc. Natl. Acad. Sci. USA 112, 4334-4339 (2015).

3. Y. Pan, F. Wang, Y. Liu, Y.-G. Yang, H. Wang, "Single-molecule-force spectroscopy study of the mechanism of interactions between TSP-1 and CD47," Sci. China Chem. 57, 1716-1722 (2014).

4. C. Boscher, J. W. Dennis, I. R. Nabi, "Glycosylation, galectins and cellular signaling," Curr. Opin. Cell Biol. 23, 383-392 (2011).

5. M. Sperandio, C. A. Gleissner, K. Ley, "Glycosylation in immune cell trafficking," Immunol. Rev. 230, 97113 (2009).

6. I. Bucior, M. M. Burger, "Carbohydrate-carbohydrate interaction as a major force initiating cell-cell recognition," Glycoconj. J. 21, 111-123 (2004).

7. S. V. Glavey, D. Huynh, M. R. Reagan, S. Manier, M. Moschetta, Y. Kawano, A. M. Roccaro, I. M. Ghobrial, L. Joshi, M. E. O'Dwyer, "The cancer glycome: Carbohydrates as mediators of metastasis," Blood Rev. 29, 269-279 (2015).

8. V. N. Tra, D. H. Dube, "Glycans in pathogenic bacteria-potential for targeted covalent therapeutics and imaging agents," Chem. Commun. 50, 4659-4673 (2014).

9. M. Dalziel, M. Crispin, C. N. Scanlan, N. Zitzmann, R. A. Dwek, "Emerging principles for the therapeutic exploitation of glycosylation," Science $\mathbf{3 4 3}$, 1235681 (2014).
10. K. Ohtsubo, J. D. Marth, "Glycosylation in cellular mechanisms of health and disease," Cell 126, 855867 (2006).

11. F. M. Goñi, "The basic structure and dynamics of cell membranes: An update of the Singer-Nicolson model," BBA. Biomembranes 1838, 1467-1476 (2014).

12. G. L. Nicolson, "The Fluid-Mosaic Model of Membrane Structure: Still relevant to understanding the structure, function and dynamics of biological membranes after more than 40 years," $B B A$. Biomembranes 1838, 1451-1466 (2014).

13. B. Truong-Quang, P. Lenne, "Membrane microdomains: From seeing to understanding," Front. Plant Sci. 5, 18 (2014).

14. S. Li, X. Zhang, W. Wang, "Selective aggregation of membrane proteins by membrane-mediated interactions," Sci. China Chem. 57, 1683-1689 (2014).

15. Y. Shan, H. Wang, "The structure and function of cell membranes examined by atomic force microscopy and single-molecule force spectroscopy," Chem. Soc. Rev. 44, 3617-3638 (2015).

16. W. Zhao, Y. Tian, M. Cai, F. Wang, J. Wu, J. Gao, S. Liu, J. Jiang, S. Jiang, H. Wang, "Studying the Nucleated Mammalian Cell Membrane by Single Molecule Approaches," PLoS ONE 9, e91595 (2014).

17. Y. Wang, J. Gao, X. Guo, T. Tong, X. Shi, L. Li, M. Qi, Y. Wang, M. Cai, J. Jiang, C. Xu, H. Ji, H. Wang, "Regulation of EGFR nanocluster formation by ionic protein-lipid interaction," Cell Res. 24, 959-976 (2014).

18. J. Wu, J. Gao, M. Qi, J. Wang, M. Cai, S. Liu, X. Hao, J. Jiang, H. Wang, "High-efficiency localization of $\mathrm{Na}^{+}-\mathrm{K}^{+}$ATPases on the cytoplasmic side by direct stochastic optical reconstruction microscopy," Nanoscale 5, 11582-11586 (2013).

19. S. K. Saka, A. Honigmann, C. Eggeling, S. W. Hell, T. Lang, S. O. Rizzoli, "Multi-protein assemblies underlie the mesoscale organization of the plasma membrane," Nat. Commun. 5, 4509-4522 (2014).

20. Y. Mechref, M. V. Novotny, "Glycomic analysis by capillary electrophoresis-mass spectrometry," Mass Spectrom. Rev. 28, 207-222 (2009).

21. J. L. Behan, K. D. Smith, "The analysis of glycosylation: A continued need for high $\mathrm{pH}$ anion exchange chromatography," Biomed. Chromatogr. 25, 39-46 (2011).

22. L. Royle, M. P. Campbell, C. M. Radcliffe, D. M. White, D. J. Harvey, J. L. Abrahams, Y.-G. Kim, G. W. Henry, N. A. Shadick, M. E. Weinblatt, "HPLC-based analysis of serum N-glycans on a 
96-well plate platform with dedicated database software," Anal. Biochem. 376, 1-12 (2008).

23. J. Zaia, "Mass spectrometry and glycomics," Omics 14, 401-418 (2010).

24. S.-C. Tao, Y. Li, J. Zhou, J. Qian, R. L. Schnaar, Y. Zhang, I. J. Goldstein, H. Zhu, J. P. Schneck, "Lectin microarrays identify cell-specific and functionally significant cell surface glycan markers," Glycobiology 18, 761-769 (2008).

25. Y. Liu, A. S. Palma, T. Feizi, "Carbohydrate microarrays: Key developments in glycobiology," Biol. Chem. 390, 647-656 (2009).

26. E. W. Adams, D. M. Ratner, H. R. Bokesch, J. B. McMahon, B. R. O'Keefe, P. H. Seeberger, "Oligosaccharide and glycoprotein microarrays as tools in HIV glycobiology: Glycan-dependent gp120/ protein interactions," Chem. Biol. 11, 875-881 (2004).

27. X.-D. Xu, H. Cheng, W.-H. Chen, S.-X. Cheng, R.-X. Zhuo, X.-Z. Zhang, "In situ recognition of cellsurface glycans and targeted imaging of cancer cells," Sci. Rep. 3 2679-2686 (2013).

28. A. Neves, H. Stöckmann, Y. Wainman, J. Kuo, S. Fawcett, F. J. Leeper, K. M. Brindle, "Imaging cell surface glycosylation in vivo using 'double click' chemistry," Bioconjugate Chem. 24, 934-941 (2013).

29. D. R. Whelan, T. D. Bell, "Super-Resolution singlemolecule localization microscopy: Tricks of the trade," J. Phys. Chem. Lett. 6, 374-382 (2015).

30. E. F. Fornasiero, F. Opazo, "Super-resolution imaging for cell biologists," BioEssays: News Rev. Mol. Cell. Develop. Biol. 37, 436-451 (2015).

31. S. W. Hell, J. Wichmann, "Breaking the diffraction resolution limit by stimulated emission: Stimulatedemission-depletion fluorescence microscopy," Opt. Lett. 19, 780-782 (1994).

32. K. I. Willig, S. O. Rizzoli, V. Westphal, R. Jahn, S. W. Hell, "STED microscopy reveals that synaptotagmin remains clustered after synaptic vesicle exocytosis," Nature 440, 935-939 (2006).

33. S. W. Hell, "Far-field optical nanoscopy," Science 316, 1153-1158 (2007).

34. M. G. Gustafsson, "Nonlinear structured-illumination microscopy: Wide-field fluorescence imaging with theoretically unlimited resolution," Proc. Natl Acad. Sci. USA 102, 13081-13086 (2005).

35. M. J. Rust, M. Bates, X. Zhuang, "Sub-diffractionlimit imaging by stochastic optical reconstruction microscopy (STORM)," Nat. Methods 3, 793-796 (2006).

36. M. Heilemann, S. van de Linde, M. Schüttpelz, R. Kasper, B. Seefeldt, A. Mukherjee, P. Tinnefeld, M. Sauer, "Subdiffraction-resolution fluorescence imaging with conventional fluorescent probes," Angew. Chem., Int. Ed. 47, 6172-6176 (2008).
37. E. Betzig, G. H. Patterson, R. Sougrat, O. W. Lindwasser, S. Olenych, J. S. Bonifacino, M. W. Davidson, J. Lippincott-Schwartz, H. F. Hess, "Imaging intracellular fluorescent proteins at nanometer resolution," Science 313, 1642-1645 (2006).

38. S. T. Hess, T. P. Girirajan, M. D. Mason, "Ultrahigh resolution imaging by fluorescence photoactivation localization microscopy," Biophys. J. 91, 4258-4272 (2006).

39. S. Rocha, H. De Keersmaecker, H. Uji-i, J. Hofkens, H. Mizuno, Photoswitchable fluorescent proteins for superresolution fluorescence microscopy circumventing the diffraction limit of light, Methods Mol. Bio. 1076, 793-812 (2014).

40. S. van de Linde, S. Aufmkolk, C. Franke, T. Holm, T. Klein, A. Löschberger, S. Proppert, S. Wolter, M. Sauer, "Investigating cellular structures at the nanoscale with organic fluorophores," Chem. Biol. 20, 8-18 (2013).

41. S. van de Linde, A. Löschberger, T. Klein, M. Heidbreder, S. Wolter, M. Heilemann, M. Sauer, "Direct stochastic optical reconstruction microscopy with standard fluorescent probes," Nat. Protoc. 6, 991-1009 (2011).

42. M. Bates, B. Huang, G. T. Dempsey, X. Zhuang, "Multicolor super-resolution imaging with photoswitchable fluorescent probes," Science 317, 17491753 (2007).

43. J. Wang, J. Li, Y. Yang, M. Yang, P. R. Chen, Small-molecule labeling probes, Optical Nanoscopy and Novel Microscopy Techniques, X. Peng, Ed., Chap. 4, p. 85, CRC Press [Imprint]; Taylor \& Francis Group, Florida, United States (2014).

44. C. Uttamapinant, J. D. Howe, K. Lang, V. Beránek, L. Davis, M. Mahesh, N. P. Barry, J. W. Chin, "Genetic code expansion enables live-cell and superresolution imaging of site-specifically labeled cellular proteins," J. Am. Chem. Soc. 137, 4602-4605 (2015).

45. J. Patrick, "Click chemistry facilitates direct labelling and super-resolution imaging of nucleic acids and proteins," RSC Adv. 4, 30462-30466 (2014).

46. C. Chu, Y. Shan, H. Wang, "Application of quantum dots in biological detection," Chin. J. Appl. Chem. 31, 377-388 (2014).

47. K. Finan, B. Flottmann, M. Heilemann, Photoswitchable fluorophores for single-molecule localization microscopy, Nanoimaging: Methods and Protocols 9, 131-151 (2013).

48. M. Ormö, A. B. Cubitt, K. Kallio, L. A. Gross, R. Y. Tsien, S. J. Remington, "Crystal structure of the Aequorea victoria green fluorescent protein," Science 273, 1392-1395 (1996).

49. E. L. Snapp, "Fluorescent proteins: A cell biologist's user guide," Trends Cell Biol. 19, 649-655 (2009). 
50. S. Manley, J. M. Gillette, G. H. Patterson, H. Shroff, H. F. Hess, E. Betzig, J. Lippincott-Schwartz, "High-density mapping of single-molecule trajectories with photoactivated localization microscopy," Nat. Methods 5, 155-157 (2008).

51. H. Shroff, C. G. Galbraith, J. A. Galbraith, E. Betzig, "Live-cell photoactivated localization microscopy of nanoscale adhesion dynamics," Nat. Methods 5, 417-423 (2008).

52. M. Heilemann, E. Margeat, R. Kasper, M. Sauer, P. Tinnefeld, "Carbocyanine dyes as efficient reversible single-molecule optical switch," J. Am. Chem. Soc. 127, 3801-3806 (2005).

53. M. Bates, T. R. Blosser, X. Zhuang, "Short-range spectroscopic ruler based on a single-molecule optical switch," Phys. Rev. Lett. 94, 108101 (2005).

54. M. Sauer, A practical guide to dSTORM: Superresolution imaging with standard fluorescent probes, Far-Field Optical Nanoscopy, P. Tinnefeld, C. Eggeling, S. W. Hell, Eds., Chap. 1, pp. 65-84, Springer, Berlin, Heidelberg (2015).

55. G. Lukinavičius, K. Umezawa, N. Olivier, A. Honigmann, G. Yang, T. Plass, V. Mueller, L. Reymond, I. R. Corrêa Jr, Z.-G. Luo, "A near-infrared fluorophore for live-cell super-resolution microscopy of cellular proteins," Nat. Chem. 5, 132-139 (2013).

56. R. Wieneke, A. Raulf, A. Kollmannsperger, M. Heilemann, R. Tampé, "SLAP: Small labeling pair for single-molecule super-resolution imaging," Angew. Chem., Int. Ed. 54, 10216-10219 (2015).

57. J. Ries, C. Kaplan, E. Platonova, H. Eghlidi, H. Ewers, "A simple, versatile method for GFP-based super-resolution microscopy via nanobodies," Nat. Methods 9, 582-584 (2012).

58. M. Mikhaylova, B. M. Cloin, K. Finan, R. van den Berg, J. Teeuw, M. M. Kijanka, M. Sokolowski, E. A. Katrukha, M. Maidorn, F. Opazo, "Resolving bundled microtubules using antitubulin nanobodies," Nat. Commun. 6, 7933-7939 (2015).

59. F. Opazo, M. Levy, M. Byrom, C. Schäfer, C. Geisler, T. W. Groemer, A. D. Ellington, S. O. Rizzoli, "Aptamers as potential tools for super-resolution microscopy," Nat. Methods 9, 938-939 (2012).

60. P. J. Zessin, K. Finan, M. Heilemann, "Super-resolution fluorescence imaging of chromosomal DNA," J. Struct. Biol. 177, 344-348 (2012).

61. T. Zheng, D. Peelen, L. M. Smith, "Lectin arrays for profiling cell surface carbohydrate expression," J. Am. Chem. Soc. 127, 9982-9983 (2005).

62. J. Lescar, J.-F. Sanchez, A. Audfray, J.-L. Coll, C. Breton, E. P. Mitchell, A. Imberty, "Structural basis for recognition of breast and colon cancer epitopes Tn antigen and Forssman disaccharide by
Helix pomatia lectin," Glycobiology 17, 1077-1083 (2007).

63. A. Cibiel, D. M. Dupont, F. Ducongé, "Methods to identify aptamers against cell surface biomarkers," Pharmaceuticals. 4, 1216-1235 (2011).

64. J. Kawakami, Y. Kawase, N. Sugimoto, "In vitro selection of aptamers that recognize a monosaccharide," Anal. Chim. Acta 365, 95-100 (1998).

65. Q. Yang, I. J. Goldstein, H.-Y. Mei, D. R. Engelke, "DNA ligands that bind tightly and selectively to cellobiose," Proc. Natl. Acad. Sci. USA 95, 54625467 (1998).

66. M. M. Masud, M. Kuwahara, H. Ozaki, H. Sawai, "Sialyllactose-binding modified DNA aptamer bearing additional functionality by SELEX," Bioorg. Med. Chem. 12, 1111-1120 (2004).

67. S. Jeong, T.-Y. Eom, S.-J. Kim, S.-W. Lee, J. Yu, "In vitro selection of the RNA aptamer against the Sialyl Lewis X and its inhibition of the cell adhesion," Biochem. Biophys. Res. Commun. 281, 237-243 (2001).

68. B. Boese, K. Corbino, R. Breaker, "In vitro selection and characterization of cellulose-binding RNA aptamers using isothermal amplification," Nucleos. Nucleot. Nucl. Acids 27, 949-966 (2008).

69. S. Y. Low, J. E. Hill, J. Peccia, "DNA aptamers bind specifically and selectively to $(1 \rightarrow 3)-\beta$ $d$-glucans," Biochem. Biophys. Res. Commun. 378, 701-705 (2009).

70. G. A. Rabinovich, M. A. Toscano, S. S. Jackson, G. R. Vasta, "Functions of cell surface galectin-glycoprotein lattices," Curr. Opin. Struct. Biol. 17, 513520 (2007).

71. W. Sun, L. Du, M. Li, "Aptamer-based carbohydrate recognition," Curr. Pharm. Des. 16, 22692278 (2010).

72. J. Li, H. Wu, J. Hong, X. Xu, H. Yang, B. Wu, Y. Wang, J. Zhu, R. Lai, X. Jiang, "Odorranalectin is a small peptide lectin with potential for drug delivery and targeting," PLoS One 3, e2381 (2008).

73. Y. Konami, K. YamRmoto, T. Osawa, T. Irimura, "The primary structure of the Cytisus scoparius seed lectin and a carbohydrate-binding peptide," $J$. Biochem. 112, 366-375 (1992).

74. K. Yamamoto, Y. Konami, T. Osawa, T. Irimura, "Carbohydrate-binding peptides from several anti- $\mathrm{H}$ (O) lectins," J. Biochem. 111, 436-439 (1992).

75. K. Yamamoto, Y. Konami, T. Osawa, "Determination of the carbohydrate-binding site of Bauhinia purpurea lectin by affinity chromatography," J. Chromatogr. A 597, 221-230 (1992).

76. L. Heerze, P. Chong, G. Armstrong, "Investigation of the lectin-like binding domains in pertussis toxin using synthetic peptide sequences. Identification of a 
sialic acid binding site in the $\mathrm{S} 2$ subunit of the toxin," J. Biol. Chem. 267, 25810-25815 (1992).

77. S. H. Tam, M. T. Nakada, M. Kruszynski, W. E. Fieles, A. H. Taylor, M. Mervic, G. A. Heavner, "Structure-function studies on synthetic peptides derived from the 109-118 lectin domain of selectins," Biochem. Biophys. Res. Commun. 227, 712-717 (1996).

78. C. E. Von Seggern, R. J. Cotter, "Study of peptidesugar non-covalent complexes by infrared atmospheric pressure matrix-assisted laser desorption/ ionization," J. Mass Spectrom. 39, 736-742 (2004).

79. E. N. Peletskaya, V. V. Glinsky, G. V. Glinsky, S. L. Deutscher, T. P. Quinn, "Characterization of peptides that bind the tumor-associated ThomsenFriedenreich antigen selected from bacteriophage display libraries," J. Mol. Biol. 270, 374-384 (1997).

80. S. Hyun, J. Kim, M. Kwon, J. Yu, "Selection and syntheses of tentacle type peptides as 'artificial' lectins against various cell-surface carbohydrates," Bioorg. Med. Chem. 15, 511-517 (2007).

81. S. Hyun, E. H. Lee, J. Park, J. Yu, "Tentacle type peptides as artificial lectins against sulfated Lewis X and A," Bioorg. Med. Chem. Lett. 18, 4011-4014 (2008).

82. K. W. Boltz, M. J. Gonzalez-Moa, P. Stafford, S. A. Johnston, S. A. Svarovsky, "Peptide microarrays for carbohydrate recognition," Analyst 134, 650-652 (2009).

83. M. Sawa, T.-L. Hsu, T. Itoh, M. Sugiyama, S. R. Hanson, P. K. Vogt, C.-H. Wong, "Glycoproteomic probes for fluorescent imaging of fucosylated glycans in vivo," Proc. Natl. Acad. Sci. USA 103, 1237112376 (2006).

84. E. Saxon, C. R. Bertozzi, "Cell surface engineering by a modified staudinger reaction," Science $\mathbf{2 8 7}$, 2007-2010 (2000).

85. P. V. Chang, J. A. Prescher, M. J. Hangauer, C. R. Bertozzi, "Imaging cell surface glycans with bioorthogonal chemical reporters," J. Am. Chem. Soc. 129, 8400-8401 (2007).

86. S. J. Luchansky, S. Goon, C. R. Bertozzi, "Expanding the diversity of unnatural cell-surface sialic acids," ChemBioChem 5, 371-374 (2004).

87. T.-L. Hsu, S. R. Hanson, K. Kishikawa, S.-K. Wang, M. Sawa, C.-H. Wong, "Alkynyl sugar analogs for the labeling and visualization of glycoconjugates in cells," Proc. Natl. Acad. Sci. USA 104, 2614-2619 (2007).

88. D. H. Dube, J. A. Prescher, C. N. Quang, C. R. Bertozzi, "Probing mucin-type O-linked glycosylation in living animals," Proc. Natl. Acad. Sci. USA 103, 4819-4824 (2006).

89. M. Boyce, I. S. Carrico, A. S. Ganguli, S.-H. Yu, M. J. Hangauer, S. C. Hubbard, J. J. Kohler, C. R. Bertozzi, "Metabolic cross-talk allows labeling of Olinked $\beta$-N-acetylglucosamine-modified proteins via the N-acetylgalactosamine salvage pathway," Proc. Natl. Acad. Sci. USA 108, 3141-3146 (2011).

90. D. Rabuka, S. C. Hubbard, S. T. Laughlin, S. P. Argade, C. R. Bertozzi, "A chemical reporter strategy to probe glycoprotein fucosylation," J. Am. Chem. Soc. 128, 12078-12079 (2006).

91. S. T. Laughlin, J. M. Baskin, S. L. Amacher, C. R. Bertozzi, "In vivo imaging of membrane-associated glycans in developing zebrafish," Science 320, 664667 (2008).

92. P. V. Robinson, G. de Almeida-Escobedo, A. E. de Groot, J. L. McKechnie, C. R. Bertozzi, "Live-cell labeling of specific protein glycoforms by proximityenhanced bioorthogonal ligation," J. Am. Chem. Soc. 137, 10452-10455 (2015).

93. S. Letschert, A. Göhler, C. Franke, N. Bertleff Zieschang, E. Memmel, S. Doose, J. Seibel, M. Sauer, "Super-resolution imaging of plasma membrane glycans," Angew. Chem., Int. Ed. 53, 10921-10924 (2014).

94. H. Jiang, B. P. English, R. B. Hazan, P. Wu, B. Ovryn, "Tracking surface glycans on live cancer cells with single-molecule sensitivity," Angew. Chem., Int. Ed. 54, 1765-1769 (2014).

95. H. C. Hang, C. Yu, D. L. Kato, C. R. Bertozzi, "A metabolic labeling approach toward proteomic analysis of mucin-type O-linked glycosylation," Proc. Natl. Acad. Sci. USA 100, 14846-14851 (2003).

96. S. Stairs, A. A. Neves, H. Stöckmann, Y. A. Wainman, H. Ireland-Zecchini, K. M. Brindle, F. J. Leeper, "Metabolic glycan imaging by isonitriletetrazine click chemistry," ChemBioChem 14, 10631067 (2013).

97. J. Chen, J. Gao, J. Wu, M. Zhang, M. Cai, H. Xu, J. Jiang, Z. Tian, H. Wang, "Revealing the carbohydrate pattern on a cell surface by super-resolution imaging," Nanoscale 7, 3373-3380 (2015). 